\title{
P16 and p53 Expression in (Pre)Malignant Epidermal Tumors of Renal Transplant Recipients and Immunocompetent Individuals
}

Willeke A.M. Blokx, M.D., Elke M.G.J. de Jong, M.D., Ph.D., Peter C.M. de Wilde, D.M.D., Ph.D., Johan Bulten, M.D., Ph.D., Monique M.G.M. Link, Dirk J. Ruiter, M.D., Ph.D.,

Peter C.M. van de Kerkhof, M.D., Ph.D.

Departments of Pathology (WAMB, PCMdW, JB, MMGML, DJR) and Dermatology (EMGJdJ, PCMvdK), University Medical Center St. Radboud, Nijmegen, the Netherlands

Ultraviolet (UV) radiation is a prevailing factor implicated in the etiology of keratinocytic intraepidermal neoplasia (KIN) and squamous cell carcinomas (SCCs), as evidenced by the high frequency of UVrelated mutations in the p53 and p16 tumor suppressor genes. In renal transplant recipients (RTRs), immunosuppression is considered another important risk factor in the enhanced carcinogenesis in these patients. So far, effects of UV and immune status on p53 and p16 immunoexpression in SCCs and precursors have not been studied. The aims of this study were to assess (1) the relation between risk factors for carcinogenesis, sun exposure and immune status, and p16 or p53 expression, and (2) to assess differences in p16 and p53 expression between KINs and SCCs. Immunostaining for p16 and p53 was performed on paraffin-embedded sections of 23 low-grade KIN (LKIN) lesions, 28 high-grade KINs (HKINs), and 35 SCCs from 44 RTRs and 42 immunocompetent controls (ICIs). In 74/86 lesions (86\%), p53 was expressed, and in $63 / 86$ (76\%) lesions, p16 expression was present. Negativity for both p16 and p53 was found in 4/86 (5\%) cases, whereas combined p53/p16 staining was most prevalent (55/86 lesions, $64 \%)$. P16 staining proved independent of p53 expression $(P=.8)$, and immune status, sun exposure, and histological diagnosis (LKIN-HKIN-SCC) had no influence on this independence. Transplantation was associated with p53 expression in SCCs $(P=.02$; power $=34 \%)$ caused by higher prevalence of p53-negative SCCs in RTRs

Copyright (C) 2003 by The United States and Canadian Academy of Pathology, Inc.

VOL. 16, NO. 9, P. 869, 2003 Printed in the U.S.A.

Date of acceptance: June 5, 2003.

Address reprint requests to: Willeke A.M. Blokx, M.D., University Medical Center St. Radboud, Department of Pathology, Geert Grooteplein 24, 6525 GA Nijmegen, the Netherlands; fax: 31-243540520; e-mail: w.blokx@pathol.umcn.nl.

DOI: 10.1097/01.MP.0000084435.89035.4C than in ICIs ( $30 \%$ versus 0$)$. In HKINs, p16 was more frequently positive than in LKINs $(P=.003$; power $=49 \%)$ and SCCs $(P=.03$; power $=53 \%)$. HKINs showed more frequent transepidermal p16 and p53 staining than LKIN lesions $(P<.001$; power $\geq 99 \%)$. This study demonstrates that in KIN lesions and cutaneous SCCs, p16 expression is independent of p53 expression, and immune status, sun exposure, and histological diagnosis have no influence on this independence. Furthermore, HKIN lesions express significantly more p16 than LKINs and SCCs.

KEY WORDS: Actinic keratosis, Carcinoma, Immunosuppression, p16, p53, Renal transplant recipient, Skin, Sun.

Mod Pathol 2003;16(9):869-878

Based on epidemiological, clinical, histopathological, and molecular studies, keratinocytic intraepidermal neoplasia (KIN) is considered an early step in squamous cell carcinoma (SCC) development (1). Ultraviolet (UV) radiation is a prevailing factor implicated in the etiology of KIN and SCCs as evidenced by the high frequency of UV-related mutations in both the p53 and p16 tumor suppressor genes (2-5).

$\mathrm{p} 16^{\mathrm{INK} 4 \mathrm{~A}}$ (p16) is encoded by the INK4a/CDKN2A gene located on chromosome 9p21 (6). P16 is a cyclin-dependent kinase inhibitor that specifically blocks the activity of cyclin-dependent kinases (CDKs) CDK4 and CDK6. By binding to CDK4, p16 ${ }^{\text {INK4A }}$ can inhibit the phosphorylation of pRB with subsequent inhibition of E2F release and arrest of the cell cycle in G1 phase and suppression of cell proliferation. Functional or structural loss of p16, could therefore lead to cell cycle propagation of potential genetically damaged cells and subsequent risk of tumor development. Links between the p16 and p53 pathways are present and mediated by $\mathrm{p} 14^{\mathrm{ARF}}$, which is formed by an alternative 
reading frame in the INK $4 a$ gene, which is shared by p16 and p14 $(6,7)$. Therefore, loss of the INK $4 a$ gene disrupts two cell control pathways, one through $\mathrm{p} 16^{\mathrm{INK} 4 \mathrm{~A}} / \mathrm{CDK} 4 / 6 / \mathrm{pRB}$ and the other through $\mathrm{p} 14^{\mathrm{ARF}} / \mathrm{MDM} 2 / \mathrm{p} 53$.

P53 mutations are the most frequent genetic alteration in SCC of the skin with reported incidence of $63 \%$ in sporadic tumors and $48 \%$ in skin carcinomas of RTRs (2). The majority of mutations bear a UV signature.

UV-induced mutations of p16 have been reported in sporadic SCCs $(\leq 24 \%)(3,4)$ and SCCs of xeroderma pigmentosum patients $(33 \%)$. In the latter, statistically significant associations were found between the frequency of mutations in p53 and p16 and between the frequency of mutations in p53 and in p14 (5). These positive associations were not found for the sporadic skin carcinomas (3). Furthermore, experiments in cultured human keratinocytes have demonstrated different induction of both p16 and p53 by UVB radiation (8). All these data suggest that the p53 and p16 proteins are part of parallel pathways controlling cell cycle and response to UV DNA damage in keratinocytes.

Immunocompromised patients, such as renal transplant recipients, have a markedly increased risk of developing KINs and SCCs of the skin, especially on sun-exposed parts of the body $(9,10)$. In these patients, besides UV, immunosuppressive treatment and human papilloma virus (HPV) also are considered risk factors for the enhanced cutaneous carcinogenesis (10-12). The multiplicity of skin cancers in these patients and these cancers' more aggressive behavior, with metastases in a considerable number of patients, suggest that the SCCs and their precursors in RTRs differ from those in immunocompetent individuals (ICIs) with regard to their clinicopathological behavior, which might be reflected in different expression of cell-cycle-associated proteins.

The aims of the present clinicopathological study were as follows: (1) to assess a possible relation between two risk factors for cutaneous carcinogenesis (sun exposure and immune status) and the expression of p16 and p53 in patients with KIN and SCCs, (2) to assess whether the expression of p16 and p53 are statistically independent in SCCs and their precursors, and 3) to assess differences in p16 and p53 expression between KINs and SCCs.

\section{MATERIALS AND METHODS}

\section{Patients and Histopathology}

For this retrospective study we retrieved formalin-fixed and paraffin-embedded skin excisions of 51 KIN lesions and 35 SCCs from 44 RTRs and 42 normal ICIs out of our archival material at the Department of Pathology, University Medical Center Nijmegen St. Radboud, Nijmegen, the Netherlands.

Histology of all lesions was reviewed by the same dermatopathologist. A KIN classification was applied to all dysplastic skin lesions according to the criteria for KIN grading proposed by Cockerell in 2000 (1). In this grading system, KIN I is histologically characterized by focal atypia of basal keratinocytes of the lower one third of the epidermis; KIN II lesions demonstrate (focal) atypia of keratinocytes in the lower two thirds of the epidermis (often with hyperkeratosis and sparing or some involvement of acrotrichia and acrosyringia and/or presence of acanthosis and/or basal budding). KIN III lesions are characterized by a diffuse atypical keratinocytic proliferation involving the full thickness of the epidermis. We considered KIN I and II to be low-grade KIN (LKIN), corresponding to actinic keratoses (AKs). KIN III was considered to be highgrade intraepidermal neoplasia (HKIN), corresponding to Bowen's disease or squamous cell carcinoma in situ (13). This separation was made because clinical behavior and, subsequently, treatment of AKs is generally different from Bowen's disease.

SCCs were classified according to their most poorly differentiated region, and a subclassification in three categories of well, moderately, and poorly differentiated was used (14).

\section{Immunohistochemistry}

Immunohistochemical analysis was performed on all lesions using a standard avidin-biotinperoxidase complex system with diaminobenzidine as the chromogen. In brief, $4-\mu \mathrm{m}$-thick paraffin sections were deparaffinized, hydrated, and washed in phosphate buffered saline.

The antibodies, pretreatment, and dilutions used are listed in Table 1. For p16 immunostaining we used the $\mathrm{p} 16^{\mathrm{INK} 4 \mathrm{~A}} \mathrm{Ab}-4$, clone $16 \mathrm{PO} 4$ or JC2 (Neomarkers, Fremont, CA). With this antibody we have

TABLE 1. Antibodies with Used Dilution, Pretreatment, Incubation Time, and Temperature

\begin{tabular}{lllll}
\hline \multicolumn{1}{c}{ Antibody } & Antigen & \multicolumn{1}{c}{ Source } & Dilution & Temperature \\
\hline DO7 & p53 & Neomarkers, Fremont, CA & $1: 400$ & Microwave \\
P16 ${ }^{\text {INK4 }} \mathrm{Ab}-4$ & aa 1-32 of & Neomarkers & 1:100 & Microwave \\
Clone 16PO4 or JC2 & p16 ${ }^{\text {INK4 }}$ & & & \\
\hline
\end{tabular}


experience in (pre)malignant lesions of the uterine cervix with comparable results, as reported in literature $(15,16)$. Furthermore, this is one of the few p16 antibodies with a known epitope (aal-32 of p16), whereas the epitope has not yet been determined for most other p16 antibodies.

After incubation with primary antibodies, sections were incubated for 30 minutes with biotinylated horse anti-mouse antibody (1:200, Vector Laboratories, Burlingame, CA), followed by 45 minutes' incubation with avidin-biotin complex (1:50, Vector Laboratories).

Sections were counterstained with Mayer's hematoxylin.

\section{Quantification of Immunohistochemical Results}

Immunoreactivity was scored semiquantitatively: 0 (negative), $1+(\leq 10 \%$ of lesional cells positive), $2+(10-50 \%$ of lesional cells positive $)$, or $3+(>50 \%$ lesional cells positive).

In addition, in positive-staining KIN lesions, localization of p16 and p53 immunoreactivity in the epidermal cell layers was assessed: $0=$ only basal layer positivity, 1 = positivity confined to basal one third of epidermis, 2 = positivity confined to basal two thirds of epidermis, or 3 = transepidermal positive staining.

Scoring was performed without knowledge of patient history.

\section{Statistics}

The association between the presence of p16and p53-expression was assessed with $2 \times 2$ contingency tables. The presence of p16 and p53 expression are statistically independent if the conditional odds ratios between p16 and p53 expression do not differ significantly from 1 . On the basis of the two presently studied risk factors (sun exposure and immune status), four risk groups were discerned, and on the basis of the histological diagnosis, patients were divided in three groups (LKIN, HKIN, and SCC). It may be possible that the association between the presence of p16 and p53 expression depends on the risk factors and/or the severity of the skin lesion. Therefore, analyses of stratified $2 \times 2$ tables were performed. The associations between the presence of p16 and p53 expression for the separate strata were tested with Fisher's exact test. The Breslow-Day statistic was used to test whether the associations, defined in terms of conditional odds ratios, are equal for the different strata (testing the homogeneity of odds ratios). The Mantel-Haenszel estimator was used as a common odds ratio for the several strata. This estimator was used to test whether the estimated common odds ratio differed significantly from 1 . If the Breslow-
Day test for homogeneity of odds ratios and Mantel-Haenszel test for conditional independence resulted in $P$ values of $>.05$, we concluded that the presence of p16 expression is statistically independent on the expression of p53 and that this independence is not influenced by sun exposure and immune status or histological diagnosis.

The difference between LKIN, HKIN, and SCC with regard to the extent of p53 and p16 expression in relation to the risk factors were analyzed by means of $I \times J$ two-way contingency tables. The same analyses were also used to assess the differences between well, moderately, and poorly differentiated SCCs with regard to the extent of p53 and p16 expression in relation to the two risk factors. In these analyses, significance was set at $P \leq .05$.

The SPSS exact tests, available in SPSS 10.0 for Windows, were used instead of large-sample approximations to obtain the exact $P$ values. Detailed information concerning the aforementioned statistical procedures is given in the literature (17).

Because of relatively small patient numbers, power analysis was performed using Power and Precision 2 (http://www.Power-Analysis.com). Fisher's exact method or the Casagrande and Pike method (approximation of Fisher's exact test) was used to compute power for the Fisher's exact tests performed.

\section{RESULTS}

\section{Patients}

Table 2 summarizes the data from all analyzed lesions.

Lesions from the head and neck region, hands, and forearms were considered as sun exposed: 65 of the examined 86 lesions came from sun-exposed sites $(75 \%)$; 18 cases $(21 \%)$, from nonexposed sites; and in 3 cases, the localization was unknown (4\%).

\section{Dependency of p16 Expression on p53 Expression in Relation to the Four Combinations of the Two Studied Risk Factors (Sun Exposure and Immune Status)}

The frequencies of cases with regard to the expression of p16 and p53 in the four different risk categories are given in Table 3 for 83/86 lesions (of three lesions, localization and therefore sun expo-

\begin{tabular}{|c|c|c|c|}
\hline $\begin{array}{c}\text { All cases } \\
\mathrm{n}=86\end{array}$ & M:F & $\begin{array}{c}\text { Age } \\
(\text { mean } \pm \mathrm{SD})\end{array}$ & Lesion Type and Number \\
\hline RTRs n $=44$ & $24: 20$ & $55.3 \pm 9.3$ & $\begin{array}{l}\text { LKIN } n=10 \\
\text { HKIN } n=14 \\
\text { SCC } n=20\end{array}$ \\
\hline ICIs $n=42$ & $21: 21$ & $72.5 \pm 11.4$ & $\begin{array}{l}\text { LKIN } n=13 \\
\text { HKIN } n=14 \\
\text { SCC } n=15\end{array}$ \\
\hline
\end{tabular}


TABLE 3.A. The Four Expression Patterns for p16 and p53 in Relation to the 4 Combinations of the 2 Studied Risk Factors, Transplantation Status and Sun Exposure, in 83 of the 86 Patients (in 3, specimen localization of the lesion was unknown)

\begin{tabular}{|c|c|c|c|c|c|c|}
\hline Risk Factors & $\begin{array}{l}\text { P16-/p53- No. } \\
\text { (percentage) }\end{array}$ & $\begin{array}{l}\text { P16-/p53+ No. } \\
\text { (percentage) }\end{array}$ & $\begin{array}{l}\mathrm{P} 16+/ \mathrm{p} 53-\mathrm{No} . \\
\quad \text { (percentage) }\end{array}$ & $\begin{array}{l}\mathrm{P} 16+/ \mathrm{p} 53+\mathrm{No} . \\
\quad \text { (percentage) }\end{array}$ & $\begin{array}{l}\text { Fisher Test Two-Tailed } \\
\text { P-Value }\end{array}$ & $\begin{array}{c}\text { Power } \\
\text { (Casagrande and Pike) }\end{array}$ \\
\hline Sun $-/$ RTR $-(\mathrm{N}=9)$ & $1(11)$ & $2(22)$ & 0 & $6(67)$ & .3 & $20 \%$ \\
\hline Sun-/RTR+ $(\mathrm{N}=9)$ & 0 & $1(11)$ & $2(22)$ & $6(67)$ & $>.9$ & $48 \%$ \\
\hline Sun $+/$ RTR $-(\mathrm{N}=32)$ & $1(3)$ & $9(28)$ & $5(16)$ & $17(53)$ & .6 & $61 \%$ \\
\hline Sun $+/$ RTR $+(\mathrm{N}=33)$ & $2(6)$ & $7(21)$ & $7(21)$ & $17(52)$ & $>.9$ & $72 \%$ \\
\hline Total $(\mathrm{N}=83)$ & $\mathrm{N}=4(5)$ & $\mathrm{N}=19(23)$ & $\mathrm{N}=14(17)$ & $\mathrm{N}=46(55)$ & .8 & $96 \%$ \\
\hline
\end{tabular}

sure were unknown). The Fisher's exact test disclosed that in each of the four different risk groups, the expression of p16 and p53 is statistically independent. The Mantel-Haenszel test disclosed that the odds ratios in the four risk groups did not significantly differ from $1(P=.82)$, and the BreslowDay test disclosed that the four odds ratios for these risk groups did not differ significantly $(P=.32)$.

Therefore, the expression of p16 and p53 are conditionally independent and homogeneous for the four risk groups examined in this study.

\section{Dependency of p16 Expression on p53}

Expression in Relation to Histological Diagnosis (of LKIN, HKIN, and SCC)

The frequencies of the 4 different expression patterns of p16 and p53 for patients with KIN and SSC are given in Table 4 . This table discloses that absence of both p16 and p53 was only present in 4/86 (5\%) specimens, whereas combined expression of p53 and p16 was most prevalent in 48/86 (56\%) cases.

The Fisher's exact test disclosed that in low-grade KIN, high-grade KIN, and SCC, the expressions of p16 and p53 are statistically independent. The MantelHaenszel test disclosed that the odds ratios in these three diagnostic groups did not significantly differ from $1(P=.6)$, and the Breslow-Day test disclosed that the three odds ratios for LKIN, HKIN, and SCC did not differ significantly $(P=.62)$. Therefore, the expression of p53 and p16 are conditionally independent and homogeneous for LKIN, HKIN, and SCCs (also see Fig. 1 for illustration of immunohistochemical staining combinations for p16 and p53).

\section{Extensiveness of the p16 Expression in KINs and SCCs}

P16 staining was cytoplasmic or was both cytoplasmic and nuclear. We considered both strong cytoplasmic staining as well as nuclear staining to be positive reaction, in analogue to previous studies on the uterine cervix $(15,16,18)$. Normal epidermis showed only positive staining in melanocytes, with absent staining in keratinocytes (Fig. 2A). In total, $63 / 86(73 \%)$ lesions were p16 positive, and 23/86, negative $(27 \%$; Table 5$)$. In our series, none of the lesions showed exclusive nuclear staining. The combined nuclear and cytoplasmic p16 staining was most prevalent and was present in 54 cases (63\%). Only nine cases (10\%) showed exclusively cytoplasmic staining (three LKIN lesions and six SCCs).

Figure 3, A-B, illustrates the percentage of p16positive-stained lesional cells in LKIN, HKIN, and SCCs for RTRs and ICIs, respectively. Statistical analysis disclosed no significant differences between ICIs and RTRs concerning the frequencies of the four p16 staining categories for $\operatorname{LKIN}(P=.22$; power $=68 \%)$, HKIN $(P=.46$; power $>66 \%)$, and SCC $(P=.44$; power $=74 \%)$.

In Figure 3E, the extensiveness of p16 expression in well, moderately, and poorly differentiated SCC, pooled over ICIs and RTRs, is given. The differences between the well, moderately, and poorly differentiated tumors were not significant $(P=.26$; power $=66 \%$ ).

Table 5 shows for KIN lesions the epidermal localization of p16 staining and discloses that HKIN lesions were more frequently positive than were LKIN lesions $(P=.003$; power $=49 \%)$ and SCCs $(P=.03$; power $=53 \%)$. Furthermore, strongly significant, more frequent transepidermal p16 staining was present in HKIN compared with in LKIN $(P<.001$; power $=100 \%)$; epidermal localization of p16 staining proved strongly correlated with KIN grade $(r=.83 ; P<.001$; power $=100 \%$ ).

In all p16 expressing HKIN lesion, a transepithelial expression was observed, whereas in only 3 of 13

TABLE 4.A. The Expression Patterns for p16 and p53 in Relation to Lesion Type for the Whole Group of 86 Patients

\begin{tabular}{|c|c|c|c|c|c|c|}
\hline Lesion Type & $\begin{array}{l}\text { P16-/p53- No. } \\
\quad \text { (percentage) }\end{array}$ & $\begin{array}{l}\mathrm{P} 16-/ \mathrm{p} 53+\text { No. } \\
\quad \text { (percentage) }\end{array}$ & $\begin{array}{l}\mathrm{P} 16+/ \mathrm{p} 53-\mathrm{No} . \\
\quad(\text { percentage })\end{array}$ & $\begin{array}{l}\mathrm{P} 16+/ \mathrm{p} 53+\mathrm{No} . \\
\quad(\text { percentage })\end{array}$ & $\begin{array}{c}\text { Fisher Test Two-Tailed } \\
P \text {-Value }\end{array}$ & $\begin{array}{c}\text { Power } \\
\text { (Casagrande and Pike) }\end{array}$ \\
\hline LKIN (N = 23) & $2(9)$ & $8(35)$ & $5(22)$ & $8(35)$ & .4 & $20 \%$ \\
\hline HKIN (N = 28) & 0 & $2(7)$ & $6(21)$ & $20(71)$ & $>.9$ & $68 \%$ \\
\hline $\operatorname{SCC}(\mathrm{N}=35)$ & $2(6)$ & $9(26)$ & $4(11)$ & $20(57)$ & $>.9$ & $76 \%$ \\
\hline Total & $\mathrm{N}=4(\%)$ & $\mathrm{N}=19(22)$ & $\mathrm{N}=15(17)$ & $\mathrm{N}=48(56)$ & .8 & $98 \%$ \\
\hline
\end{tabular}

The Fisher exact test disclosed that in LKIN, HKIN and SCC the expression of p16 and p53 are statistically independent. 

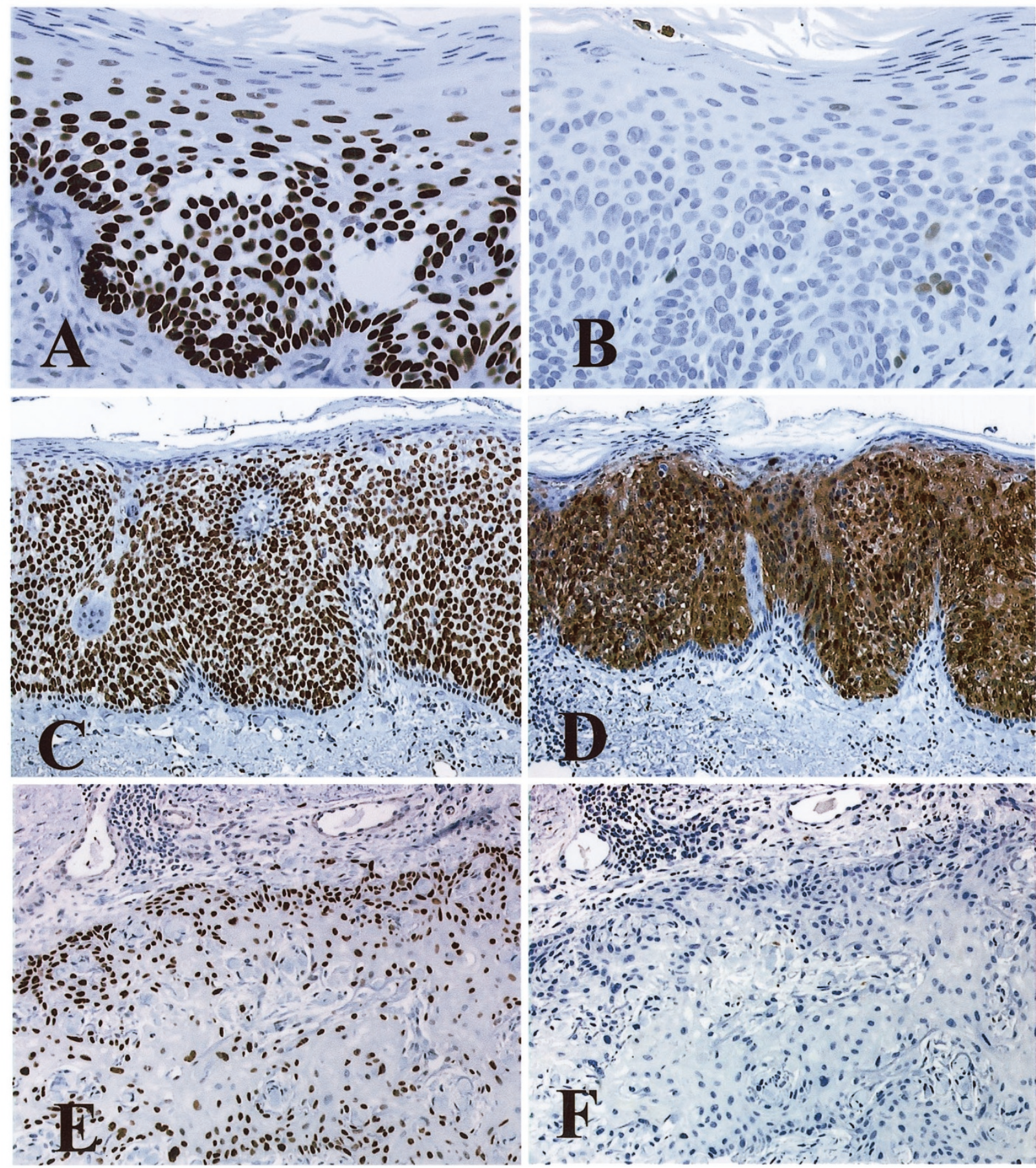

FIGURE 1. A-B, positive p53 (A) with absent $\mathrm{p} 16^{\mathrm{INK} 4 \mathrm{~A}}$ (B) expression in KIN II of an immunocompetent control patient. P53 staining is restricted to the dysplastic basal two thirds of the epidermis. C-D, strong transepidermal expression of p53 (C, nuclear staining) and p16 ${ }^{\mathrm{INK} 4 \mathrm{~A}}$ (D), nuclear and cytoplasmic) in a KIN III lesion (HKIN) of an immunocompetent patient. E-F, positive nuclear p53 (E) and absent p16 (F) staining in a squamous cell carcinoma of an immunocompetent individual.

LKIN lesions (23\%) was such an expression found. The LKIN lesions with a transepithelial expression were morphologically classified as moderate dysplasia/KIN II. In HKIN lesions, p16 staining was continuously and diffusely present throughout all epidermal layers, with sharp demarcation between the normal and dysplastic epidermis (Fig. 2, C-E).
Extensiveness of the p53 Expression in KINs and SCCs

P53 staining was nuclear. Normal epidermis showed only slight nuclear staining in a few scattered basal keratinocytes.

In total, $74 / 86$ lesions $(86 \%)$ were p53 positive and 12/86 (14\%) were p53 negative (Table 6). 


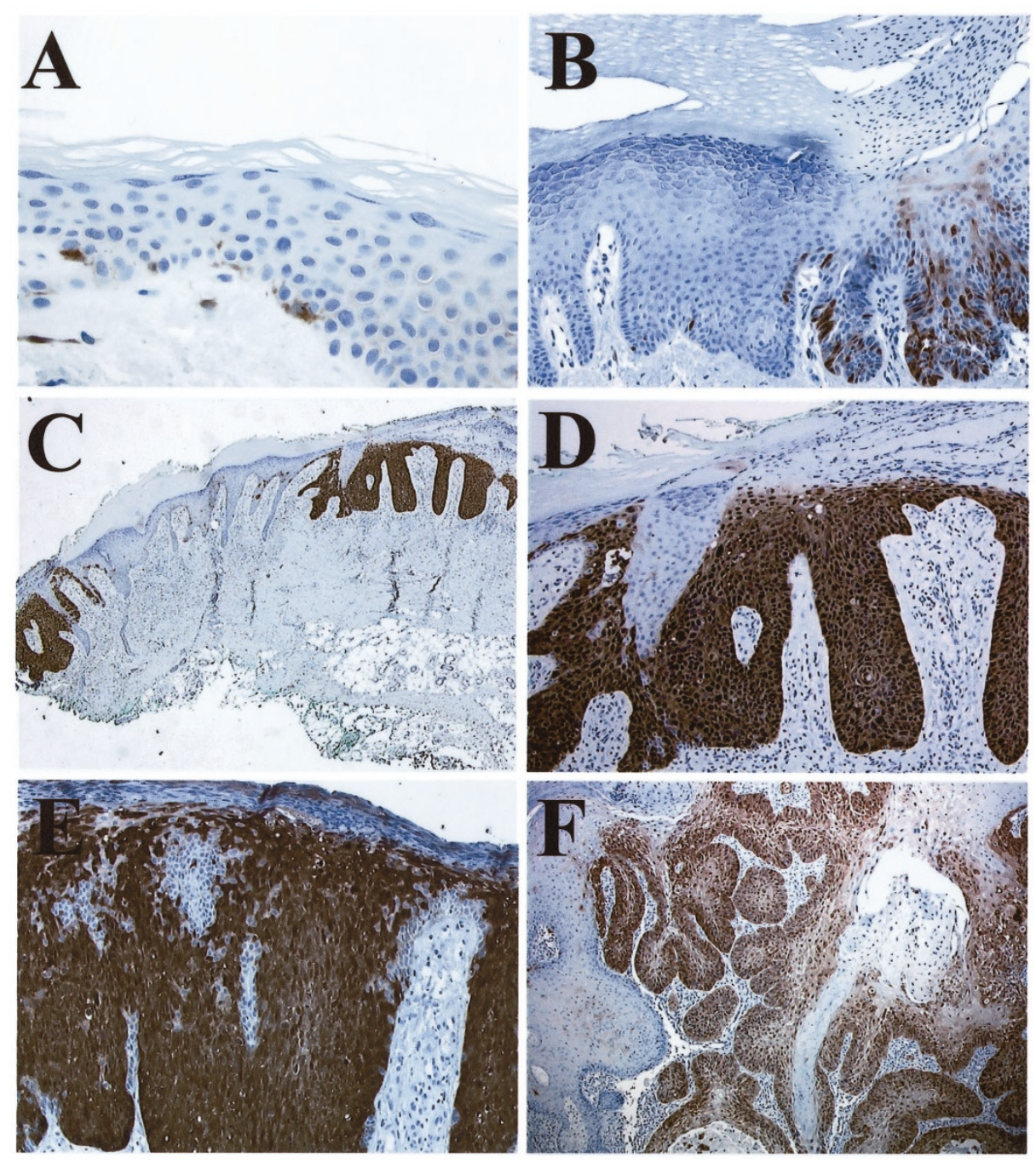

FIGURE 2. P16 expression patterns in normal skin (A) with only dendritic melanocytes staining positive; in LKIN of renal transplant recipient (RTR) showing more focal p16 staining in the basal two thirds of the epidermis (B); and in HKIN of RTR with diffuse and strong, nuclear and cytoplasmic transepidermal p16 staining, with sharp demarcation from normal skin (C-E), and in an SCC of RTR (7).

TABLE 5. P16 Expression in LKIN, HKIN, and SCCs in the Patient Group as a Whole

\begin{tabular}{|c|c|c|c|c|}
\hline & LKIN N $=23$ & HKIN N $=28$ & $\operatorname{SCC} \mathrm{N}=35$ & Total $\mathrm{N}=86$ \\
\hline P16 negative & $10(43 \%)$ & $2(7 \%)$ & $11(31 \%)$ & $23(27 \%)$ \\
\hline P16 cytopl positive & $3(13 \%)$ & 0 & $6(17 \%)$ & $9(10 \%)$ \\
\hline P16 combined nucl. \& cytopl positive & $10(43 \%)$ & $26(93 \%)$ & $18(51 \%)$ & $54(63 \%)$ \\
\hline Basal 1/3 & 5 & 0 & & \\
\hline Basal 2/3 & 5 & 0 & & \\
\hline Transepidermal & 3 & 26 & & \\
\hline
\end{tabular}

In KIN lesions further specified by location. As for p53 (Table 6), strongly significant more frequent transepidermal staining is present in HKIN compared to LKIN $(P<.001)$.

Percentages of p53-positive lesional cells in LKIN, HKIN, and SCCs for the RTRs and the ICIs, respectively, are illustrated in Figure 3, C-D. For LKIN and HKIN no significant differences were found between ICIs and RTRs with regard to the frequencies of the four p53-immunostaining categories $(P \geq 0.65$; power $=72 \%$ and $61 \%$, respectively). Only for SCCs was a significant difference in the prevalence of p53 positivity present between RTRs and ICIs $(P=.02$; power $=34 \%)$, due to a significantly higher frequency of SCC without p53 expression in RTR $(6 / 20=30 \%)$ than in ICI $(0 / 15=0 \%)$. The extensiveness of p53 expression in SCC was highly associated with grade of the SCC. The percentage of SCCs with $>50 \%$ positive lesional cells increased with decrease in differentiation grade (Fig. 3F). In well, moderately, and poorly differentiated SCCs, respectively, $14 \%$, $68 \%$, and $89 \%$ of the tumors showed $>50 \%$ positive tumor cells. The prevalence of well, moder- 


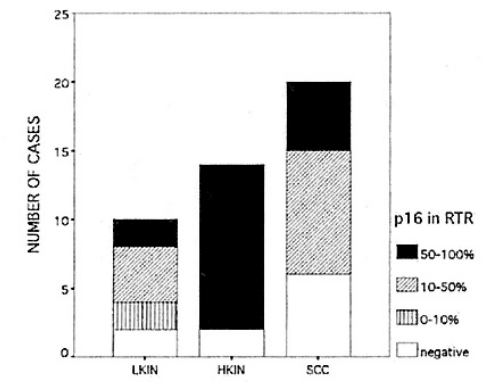

C

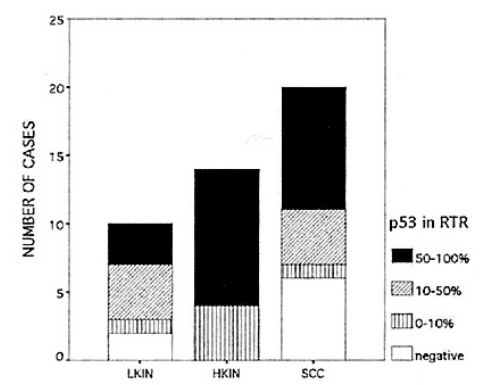

$\mathbf{E}$

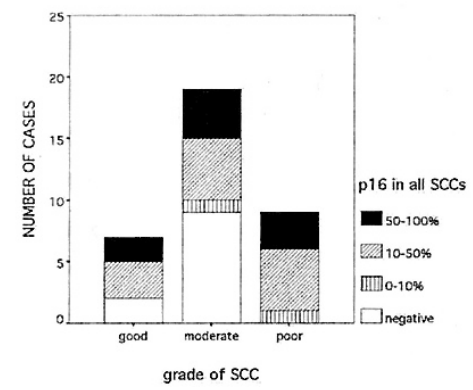

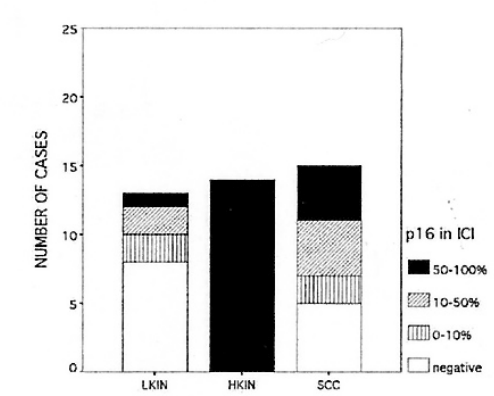

D

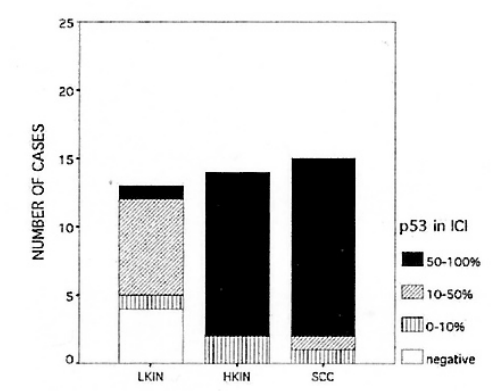

$\mathbf{F}$

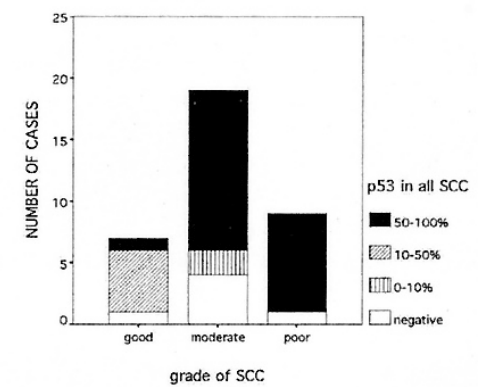

FIGURE 3. Percentages of p16- (A, B) and p53- (C, D) positive lesional cells in LKIN, HKIN, and SCCs for the RTRs and the ICIs, respectively. In E and $\mathbf{F}$, the percentages of positive lesional cells for p16 and p53, respectively, are given in all combined SCCs, divided into well, moderately, and poorly differentiated tumors.

ately, and poorly differentiated SCCs in ICIs and RTRs was not significantly different $(P=.26$; power $=66 \%$ ). Therefore, the above-described significantly higher frequency of p53-negative SCCs in RTRs is not attributable to different prevalence of tumor grades between both groups.

As for p16, in KIN lesions, strongly significant, more frequent transepidermal p53 staining was present in HKIN lesions compared with in LKIN lesions $(P<.001$ : Table 6; power $=99 \%)$. Transepithelial staining was observed in 22/28 (79\%) of p53-positive HKIN, and in only $1 / 17$ (6\%) of p53positive LKIN lesions. Absence of p53 staining was exclusively found in LKIN, and p53 expression was positively correlated with KIN grade $(r=.42 ; P<$ .001 ; power $=43 \%$ ).
TABLE 6. P53 Expression in LKIN, HKIN, and SCCs in the Patient Group as a Whole, Specified by Location in KIN Lesions

\begin{tabular}{lcccc}
\hline & LKIN & HKIN & SCC & Total \\
& $\mathrm{N}=23$ & $\mathrm{~N}=28$ & $\mathrm{~N}=35$ & $\mathrm{~N}=86$ \\
\hline P53 negative & $6(26 \%)$ & $0(0 \%)$ & $6(17 \%)$ & $12(14 \%)$ \\
P53 positive & $17(74 \%)$ & $28(100 \%)$ & $29(83 \%)$ & $74(86 \%)$ \\
Basal layer & 1 & 6 & & \\
Basal 1/3 & 8 & 0 & & \\
Basal 2/3 & 7 & 0 & & \\
Transepidermal & 1 & 22 & & \\
\hline
\end{tabular}

Strongly significant more frequent transepidermal staining is present in HKIN compared to LKIN $(P<.001)$.

\section{DISCUSSION}

The present study is the first regarding immunohistochemical expression patterns of p16 in KIN and SCCs of RTRs. RTRs develop multiple HPV- 
induced warts, AKs, and nonmelanoma skin cancers, especially on sun-exposed areas, which are directly related to the extent and duration of immunosuppression (19-24). In these immunosuppressed patients, it is thought that DNA repair and apoptotic mechanisms are abrogated by UV and HPVs and, along with the altered immune surveillance of these patients, might allow progression of benign HPV epidermal lesions to malignancy.

In this study we show that both p16 as well as p53 are frequently overexpressed in KIN and SCCs of both RTRs and ICIs and that the expression of p16 is independent of p53 expression. This independence on the protein level parallels previous molecular findings in sporadic SCCs, in which p16 and p53 mutations proved to be independent events (3). Recently, it was also demonstrated that the p16 and p53 proteins were differently modulated by UV depending on the dose and regimen (8). These latter in vitro results strongly infer that both proteins are part of parallel pathways controlling response of keratinocytes to UV DNA damage. In our clinicopathological study, we found that the expression of p16 and p53 in skin lesions for sun-exposed and non-sun-exposed areas of the body did not differ, suggesting that the p16-p53 parallelism is not influenced by UV radiation.

In addition, we found that p16 and p53 expression were conditional independent of RTR status. We found that combined p16-p53 expression was most prevalent (46/86 lesions, 56\%). Together, these observations suggest that both p16 and p53 are strongly involved in epidermal carcinogenesis but that expression levels of both proteins are independent of etiologic factors (sun exposure and immune status). Therefore, RTRs seem to use comparable pathways in skin cancer development as ICIs despite differences in immune status, but their susceptibility and rate of development of SCCs might well be determined by the deficient immune system.

Immune status was associated with p53 expression in SCCs $(P=.02)$ because of a higher prevalence of p53-negative cases in RTRs compared with in ICIs (30\% versus 0 ). Therefore, p53 seems differently involved in late stages of epidermal neoplasia in transplant recipients. Although in the present study, we did not perform PCR analysis for HPV status, one could speculate that the higher incidence of p53-negative tumors in RTRs suggests involvement of (oncogenic) HPV in the RTR group. In cervical lesions, p53 is inactivated because of the transforming activities of the E6 viral oncoprotein of the mucosal high-risk HPV types, for instance, HPV16 (25). Overexpression of p16 in the (pre)malignant lesions of the uterine cervix is assumed to be the consequence of the inactivation of pRb by E7 of high-risk HPV types $(15,18)$. So far, the knowl- edge on transforming properties of E6 and E7 of cutaneous HPV types is limited. In contrast to cervical cancer, in which high-risk HPV DNA becomes integrated in the host genome, in NMSCs containing presumed oncogenic HPV types, HPV integration is rare, and it was shown that E6 oncoprotein of, for instance, the cutaneous epidermodysplasia verruciformis-associated HPV38 could not degrade p53 protein $(26,27)$. Surprisingly, HPV 38 E6/E7positive cells also did not express p16, in contrast to HPV 16 E6/E7-positive cells, whereas both HPV 38 and HPV 16 E7 had similar biological properties, and both were able to inactivate pRb (26). We were unable to demonstrate statistical linkage of the combined staining pattern with p53 negativity and p16 positivity (indicative of oncogenic HPV involvement) to the RTR population $(P=.26)$. Therefore, based on the immunoexpression profiles for p53 and p16 presently found, and because of the incomplete knowledge of the interactions of cutaneous E6 and E7 proteins with p53 and p16, the role of HPV in cutaneous carcinogenesis in RTRs remains speculative.

So far only a few studies have evaluated p16 immunoexpression in cutaneous SCCs and precursors. Hodges and Smoller (28) described p16 positivity in all 10 AKs, SCC in situ lesions, and SCCs studied, with the use of a different antibody (G175405; Pharmingen, San Diego, CA) but a comparable scoring system, although cellular localization (either cytoplasmic and/or nuclear staining) was not further specified. Transepidermal staining was only present in SCC in situ, comparable to our results in HKINs. In contrast, we found statistically significantly more p16 positivity in HKIN lesions (93\%) than in LKIN and SCCs (57\% and 69\%, respectively, $P=.003$ and $P=.03$ ). Mortier et al. (29) found p16 expression in $66 \%$ of AKs and only $10 \%$ of SCCs, without specification of the used scoring method. In contrast to our data and those of Hodges and Smoller (28), those investigators also describe p16 expression in normal skin with the use of their antibody (G175-405, Pharmingen).

Combined mutation analysis with immunohistochemistry for p16 so far has been performed only by Chang et al. (30), albeit on a very small number of SCCs (6 cases): two of six SCCs showed positive nuclear staining for p16 (anti-p16 antibody, C-20; Santa Cruz Biotechnology), and four of six SCCs were negative. In all cases, wild type p16 was present and no mutations could be demonstrated, implicating that immunohistochemical findings cannot directly be correlated to underlying molecular alterations. These conflicting results with respect to p16 staining in SCCs of skin and precursors seem related to usage of different p16 antibodies, often-small patient numbers, and different immunohistochemical scoring methods. The question 
arises as to what these different p16 antibodies actually recognize.

In imitation of immunohistochemical studies on p16 expression in (pre)malignant lesions of the uterine cervix, we considered both nuclear and strong cytoplasmic p16 staining to be a positive reaction $(15,16,18)$. Yet we have to admit that up until now, the significance of cytoplasmic p16 staining has remained unclear, and conflicting data have been presented with respect to the specificity of cytoplasmic p16 staining in adenocarcinomas $(31,32)$. In our series, none of the lesions showed exclusive nuclear staining; the combined nuclear and cytoplasmic p16 staining was most prevalent: 54 cases (63\%) and only 9 cases (10\%) showed exclusively cytoplasmic staining (3 LKIN lesions and 6 SCCs). When considering only the cases with combined staining to be 16 positive, statistical analysis disclosed no effects on the presented conclusions (data not shown).

In conclusion, according to our data, squamous cell carcinogenesis in RTRs seems to occur along comparable pathways as in ICIs, with independent parallel involvement of both the p53 and the p16 pathway. The present study demonstrates that p53 and p16 immunoexpression are conditionally independent of the two studied risk factors, transplantation status and sun exposure. The expression of p16 proved conditionally independent of the p53 expression in KIN and SCCs, and immune status and sun exposure had no influence on this conditional independence. Future studies combining immunohistochemistry and molecular data are needed to elucidate the exact role of p16 in epidermal carcinogenesis.

\section{REFERENCES}

1. Cockerell CJ. Histopathology of incipient intraepidermal squamous cell carcinoma ("actinic keratosis"). J Am Acad Dermatol 2000;42(1 Pt 2):11-7.

2. McGregor JM, Berkhout RJ, Rozycka M, ter Schegget J, Bouwes-Bavinck JN, Brooks L, et al. p53 mutations implicate sunlight in post-transplant skin cancer irrespective of human papillomavirus status. Oncogene 1997;15(14):1737-40.

3. Soufir N, Moles JP, Vilmer C, Moch C, Verola O, Rivet J, et al. P16 UV mutations in human skin epithelial tumors. Oncogene 1999;18(39):5477-81.

4. Kubo Y, Urano Y, Matsumoto K, Ahsan K, Arase S. Mutations of the INK4a locus in squamous cell carcinomas of human skin. Biochem Biophys Res Commun 1997;232(1):38-41.

5. Soufir N, Daya-Grosjean L, de La Salmoniere P, Moles JP, Dubertret L, Sarasin A, et al. Association between INK4a-ARF and p53 mutations in skin carcinomas of xeroderma pigmentosum patients. J Natl Cancer Inst 2000;92(22):1841-7.

6. Ruas M, Peters G. The p16ink4a/CDKN2A tumor suppressor and its relatives. Biochim Biophys Acta 1998;1378:F115-77.

7. de Gruijl FR, van Kranen HJ, Mullenders LHF. UV-induced DNA. Damage, repair, mutations and oncogenic pathways in skin cancer. J Photochem Photobiol B 2001;63:19-27.

8. Chazal M, Marionnet C, Michel L, Mollier K, Dazard JE, Della Valle $\mathrm{V}$, et al. P16(INK4A) is implicated in both the immedi- ate and adaptative response of human keratinocytes to UVB irradiation. Oncogene 2002;21(17):2652-61.

9. Berg D, Otley CC. Skin cancer in organ transplant recipients: epidemiology, pathogenesis, and management. J Am Acad Dermatol 2002;47(1):1-20.

10. Euvrard S, Kanitakis J, Pouteil Noble C, Dureau G, Touraine JL, Faure M, et al. Comparative epidemiologic study of premalignant and malignant epithelial cutaneous lesions developing after kidney and heart transplantation. J Am Acad Dermatol 1995;33(2 Pt 1):222-9.

11. Weinstein T, Korzets A, Chagnac A, Ori Y, Herman M, Zevin $\mathrm{D}$, et al. Effect of immunosuppressive therapy on DNA repair and cancer incidence in renal transplant recipients. Transplant Proc 2000;32(4):694-5.

12. de Jong-Tieben LM, Berkhout RJ, Smits HL, Bouwes Bavinck JN, Vermeer BJ, van der Woude FJ, et al. High frequency of detection of epidermodysplasia verruciformis- associated human papillomavirus DNA in biopsies from malignant and premalignant skin lesions from renal transplant recipients. J Invest Dermatol 1995;105(3):367-71.

13. Fu W, Cockerell CJ. The actinic (solar) keratosis: a 21stcentury perspective. Arch Dermatol 2003;139(1):66-70.

14. McKee PH. Pathology of the skin. 2nd ed. London: Mosby; 1999.

15. Sano T, Oyama T, Kashiwabara K, Fukuda T, Nakajima T. Expression status of p16 protein is associated with human papillomavirus oncogenic potential in cervical and genital lesions. Am J Pathol 1998;153(6):1741-8.

16. Klaes R, Friedrich T, Spitkovsky D, Ridder R, Rudy W, Petry $\mathrm{U}$, et al. Overexpression of p16(INK4A) as a specific marker for dysplastic and neoplastic epithelial cells of the cervix uteri. Int J Cancer 2001;92(2):276-84.

17. Agresti A. An introduction to categorical data analysis. New York: Wiley; 1996.

18. Keating JT, Cviko A, Riethdorf S, Riethdorf L, Quade BJ, Sun D, et al. Ki-67, cyclin E, and p16INK4 are complimentary surrogate biomarkers for human papilloma virus-related cervical neoplasia. Am J Surg Pathol 2001;25(7):884-91.

19. Webb MC, Compton F, Andrews PA, Koffman CG. Skin tumours posttransplantation: a retrospective analysis of 28 years' experience at a single centre. Transplant Proc 1997; 29(1-2):828-30.

20. Hiesse C, Rieu P, Kriaa F, Larue JR, Goupy C, Neyrat N, et al. Malignancy after renal transplantation: analysis of incidence and risk factors in 1700 patients followed during a 25-year period. Transplant Proc 1997;29(1-2):831-3.

21. Bouwes Bavinck JN, Hardie DR, Green A, Cutmore S, MacNaught A, O'Sullivan B, et al. The risk of skin cancer in renal transplant recipients in Queensland, Australia. A follow-up study. Transplantation 1996;61(5):715-21.

22. Birkeland SA, Storm HH, Lamm LU, Barlow L, Blohme I, Forsberg B, et al. Cancer risk after renal transplantation in the Nordic countries, 1964-1986. Int J Cancer 1995;60(2): 183-9.

23. Hartevelt MM, Bouwes Bavinck JN, Kootte AM, Vermeer BJ, Vandenbroucke JP. Incidence of skin cancer after renal transplantation in The Netherlands. Transplantation 1990; 49(3):506-9.

24. Jensen P, Hansen S, Moller B, Leivestad T, Pfeffer P, Fauchald P. Are renal transplant recipients on CsA-based immunosuppressive regimens more likely to develop skin cancer than those on azathioprine and prednisolone? Transplant Proc 1999;31(1-2):1120.

25. Stoler MH. Human papillomaviruses and cervical neoplasia: a model for carcinogenesis. Int J Gynecol Pathol 2000;19(1): $16-28$.

26. Caldeira S, Zehbe I, Accardi R, Malanchi I, Dong W, Giarre $\mathrm{M}$, et al. The E6 and E7 proteins of the cutaneous human 
papillomavirus type 38 display transforming properties. J Virol 2003;77(3):2195-206.

27. Majewski S, Jablonska S. Do epidermodysplasia verruciformis human papillomaviruses contribute to malignant and benign epidermal proliferations? Arch Dermatol 2002;138(5):649-54.

28. Hodges A, Smoller BR. Immunohistochemical comparison of p16 expression in actinic keratoses and squamous cell carcinomas of the skin. Mod Pathol 2002;15(11):1121-5.

29. Mortier L, Marchetti P, Delaporte E, Martin de Lassalle E, Thomas P, Piette F, et al. Progression of actinic keratosis to squamous cell carcinoma of the skin correlates with deletion of the 9p21 region encoding the p16(INK4a) tumor suppressor. Cancer Lett 2002;176(2):205-14.
30. Chang TG, Wang J, Chen LW, Hsu CY, Chang HW, Chen JS, et al. Loss of expression of the pl6 gene is frequent in malignant skin tumors. Biochem Biophys Res Commun 1997;230(1):85-8.

31. Geradts J, Hruban RH, Schutte M, Kern SE, Maynard R. Immunohistochemical p16INK4a analysis of archival tumors with deletion, hypermethylation, or mutation of the CDKN2/ MTS1 gene. A comparison of four commercial antibodies. Appl Immunohistochem Mol Morphol 2000;8(1):71-9.

32. Shiozawa T, Nikaido T, Shimizu M, Zhai Y, Fujii S. Immunohistochemical analysis of the expression of cdk4 and p16INK4 in human endometrioid-type endometrial carcinoma. Cancer 1997;80(12):2250-6. 